\title{
The role of response-reinforcer associations increases throughout extended instrumental training
}

\author{
RUTH M. COLWILL and ROBERT A. RESCORLA \\ University of Pennsylvania, Philadelphia, Pennsylvania
}

\begin{abstract}
Two experiments examined the effects of extended training on the development of responsereinforcer associations. Rats were trained by using various food reinforcers to make multiple in strumental responses. Subsequently, those reinforcers were devalued by being paired with a toxin. The presence of response-reinforcer associations was inferred from the decrease in the likelihood of a response following devaluation of its reinforcer. Such response-reinforcer associations are known to contribute to performance after moderate amounts of training. These experiments addressed the question of whether the contribution of those associations remains constant, increases, or decreases with more extended training. Experiment 1 found that even after a response had been extensively trained with one reinforcer, the substitution of a new reinforcer produced new associations between the response and that new reinforcer. After extended training, a response continued to acquire new associations with a reinforcer, as indexed by the impact of a devaluation procedure. Experiment 2 directly compared the contribution of reinforcers used extensively and moderately with the same response. It found that devaluation of the extensively used reinforcer more effectively reduced performance of the response, suggesting that the associations formed with additional training contribute to performance of the response. These experiments indicate that the contribution of response-reinforcer associations does not decrease but, instead, continues to grow throughout the course of extended instrumental training.
\end{abstract}

It is a widely held view that instrumental behaviors are initially acquired as goal-directed acts but become relatively less dependent on their original goals with extended training. This notion of behavioral autonomy has both historical (Allport, 1937; Gilhousen, 1933; Murphy, 1947; Tolman, 1933, 1948) and modern proponents (Adams \& Dickinson, 1981; Kimble \& Perlmuter, 1970). One way of stating this idea is that early in training relatively specific associations are formed between instrumental responses and their outcomes, associations that govern performance of those responses. However, when those responses are repeatedly performed, other learning develops that can support performance independently of the original goal. For instance, the repeated performance of the instrumental response in a stimulus environment might lead to the development of stimulus-response (S-R) associations, strengthened either by simple contiguity or by the subsequent occurrence of a reinforcer. As a consequence, the response might come to be elicited simply by virtue of the presentation of this stimulus. In this case, performance of the response might not be influenced by the anticipated consequences of its occurrence.

One technique that has been devised for separating such alternatives involves testing the preservation of previously

This research was supported by National Science Foundation Gran BNS 83-08176 to the University of Pennsylvania. We should like to thank Anthony Dickinson for many useful conversations. Requests for reprints should be sent to either author at the Department of Psychology, University of Pennsylvania, 3815 Walnut Street, Philadelphia, PA 19104. trained instrumental performance when the original goal has been artificially devalued. That technique has proven highly effective in identifying the presence of responsereinforcer associations after a moderate amount of instrumental training. In many cases, devaluation of the goal produces a dramatic and highly specific decrease in the likelihood of behaviors that it previously reinforced (e.g. Colwill \& Rescorla, 1985b, 1986). Such results imply an important role for response-reinforcer associations after moderate training.

Recently, Colwill and Rescorla (1985a) used that technique to assess the role of these associations after more extended training. They trained rats to make four different responses, two of which earned one reinforcer (food pellets) and two of which earned another (sucrose pellets). Each reinforcer was used to train one response extensively and another moderately. Then one reinforcer was devalued by being paired with a toxin. Finally, the animals were given two nonreinforced choice tests, one between extensively trained responses and another between moderately trained responses. In both cases, the animals displayed a strong preference for the response whose reinforcer had not been devalued. Colwill and Rescorla (1985a) interpreted this result to mean that response-reinforcer associations persisted as important determinants of behavior even after extensive training.

The experiments described here addressed a somewhat more subtle question about the relationship between amount of training and response-reinforcer associations: Does the contribution of those associations continue to grow with ad- 
ditional training? One possibility is that although responsereinforcer associations form during initial acquisition, additional training results in the superimposition of another type of learning, such as an S-R association. The previously observed sensitivity to reinforcer devaluation after extensive training would then simply reflect the persistence of response-reinforcer associations acquired early in training. Alternatively, extended training might lead to either enhanced or reduced contributions of response-reinforcer associations to performance. One might expect an enhanced contribution if extended training simply continued to strengthen the associations developed in initial acquisition. On the other hand, if extended training supported an alternative S-R association, one might expect the contribution of response-reinforcer associations to be diminished. Consequently, these experiments investigated the effects of continued training on the contribution of responsereinforcer associations to instrumental performance.

Experiment 1 approached this question by introducing new features to the reinforcer after it had been used to train an instrumental response extensively. If responsereinforcer associations continue to form beyond initial training, those new reinforcer features should become associated with the response. Such associations should be exposed by a devaluation procedure applied to those new features. In contrast, if response-reinforcer learning is confined to the initial stages of learning, reinforcer features introduced only later in training should be poorly encoded and their devaluation should have little effect.

Experiment 2 addressed the same question by directly comparing the sensitivity to reinforcer devaluation of extensively and moderately trained responses. Unlike previous experiments, that comparison was made under circumstances in which response rates were matched despite substantial differences in the amount of training. If extensive training increases the contribution of responsereinforcer associations, then extensively trained behaviors might be expected to be more sensitive to reinforcer devaluation. On the other hand, if additional training involves some other learning process, extensively trained behaviors should be no more (and perhaps even less) sensitive than moderately trained behaviors.

\section{EXPERIMENT 1}

This experiment examined whether the animal acquires new information about the reinforcer when that information is introduced only after extensive training. Animals were first given extensive training of two responses with a common reinforcer, cherry-flavored pellets. Then each response was given training with one of two other reinforcers, sucrose liquid or a Noyes food pellet. An aversion was then conditioned to one of these new reinforcers, and finally, the animals were given a nonreinforced choice test between the two responses. Differential performance of those two responses could result only if new responsereinforcer associations were formed after extensive training. Because the opportunity for this new learning was deliberately made brief in this experiment, it was important to verify that such circumstances would be adequate to produce response-reinforcer associations in the absence of prior extensive training of the responses. Consequently, each animal was also given training in which two other responses were followed by the differential reinforcers; however, those responses had only a minimal history of prior reinforcement with cherry-flavored pellets.

\section{Method}

Subjects. The subjects were 16 experimentally naive Holtzmanderived male rats about 100 days old at the start of the experiment. They were maintained in individual cages at $80 \%$ of their freefeeding weights. Water was available on an ad-lib schedule.

Apparatus. The apparatus consisted of four identical Skinner boxes measuring $22.9 \times 20.3 \times 20.3 \mathrm{~cm}$. The two end walls of the chamber were aluminum; the side walls and the ceiling were clear Plexiglas. Each chamber had a recessed food magazine in the center of one end wall. A small metal cup, measuring $1.25 \mathrm{~cm}$ in diameter and $1.5 \mathrm{~cm}$ deep, was sunk in the floor of each food magazine. To the left of the magazine was a lever, and to the right was a chain suspended from a relay mounted on the roof of the chamber. Located directly above the magazine was a nose-poke manipulandum, which consisted of an aperture, $2 \mathrm{~cm}$ wide and $1.3 \mathrm{~cm}$ deep. The back of this aperture was covered by a metal plate that operated a relay switch whenever it was depressed. Under the grid floor, toward the right of the magazine was a handle-pull manipulandum. This consisted of a short flat rod with the end bent back to form a handle. Whenever the handle was pulled upward, a relay switch was closed and a response was recorded. Access to these manipulanda could be denied by covering the lever with a metal plate, by retracting the chain through an opening in the ceiling, by inserting a jewelled light cover into the aperture of the nosepoke manipulandum, and by withdrawing the arm of the handlepull. The floor of the chamber was composed of $0.48-\mathrm{cm}$ stainless steel rods spaced $1.9 \mathrm{~cm}$ apart. Each Skinner box was enclosed in a sound- and light-resistant shell. Mounted on the inside wall of each shell was a speaker that permitted presentation of an $1800-\mathrm{Hz}$ tone measuring approximately $76 \mathrm{~dB}$ re $20 \mu \mathrm{N} / \mathrm{m}^{2}$ against a background level of $62 \mathrm{~dB}$. The outside roof of the shell supported a solenoid-operated gravity-feed system, which was connected via plastic tubing to the cup in the food magazine. Experimental events were controlled and recorded automatically by relays and microprocessors located in an adjoining room.

Procedure. All subjects received two sessions of magazine training. The first session consisted of the delivery of $1045-\mathrm{mg}$ food pellets (Formula A: P. J. Noyes) followed by 10 presentations of $0.3 \mathrm{ml}$ of $8 \%$ sucrose liquid on a variable-time (VT) 60 -sec schedule. In the second session, all subjects received 1045 -mg cherryflavored pellets (P. J. Noyes) delivered on a VT 60-sec schedule. In this and all subsequent phases, the delivery of a pellet was always signaled by a distinct click of the pellet dispenser; delivery of sucrose was accompanied by a brief $1800-\mathrm{Hz}$ tone. During this phase, the response manipulanda were not accessible to the animals.

Following magazine training, two of the four responses were reinforced extensively with the cherry-flavored pellets. For half the animals, this training was conducted on the leverpress and chainpull responses; for the remaining half, nose-poking and handlepulling were reinforced. To encourage approximately equivalent levels of responding, only one manipulandum was ever available in any single training session. Initially, each response designated for extended training was reinforced continuously until 50 reinforcers had been earned. Either chain-pulling or nose-poking was reinforced on Day 1; leverpressing or handle-pulling was reinforced on Day 2. The next day, each response was reinforced for $20 \mathrm{~min}$ 
on a variable-interval (VI) 30-sec schedule. Subjects continued to receive two $20-\mathrm{min}$ sessions per day with each of these responses reinforced on a VI $60-\mathrm{sec}$ schedule. Extensive training of these responses was conducted for 13 sessions. After the ninth session of this extensive training, the two responses that had not received any prior training were each given a session of reinforcement with the cherry-flavored pellet. Each response was reinforced until 25 reinforcers had been earned.

Following the last session of extended VI training, all subjects were given two 20-min training sessions with each of the four responses reinforced on a VI 60 -sec schedule. For one of the extensively trained and one of the moderately trained responses, the reinforcer was $8 \%$ sucrose; for the remaining two responses, it was Noyes food pellets.

The consequence of this design was that sucrose and pellets were each used to reinforce one response that had undergone extensive training with the cherry-flavored pellet and one response that had received only moderate training with that cherry-flavored reinforcer.

After completion of response training, an aversion was conditioned to either sucrose or food pellets. This conditioning was carried out in the operant chambers over five 2-day cycles. On the first day of each cycle, the to-be-conditioned reinforcer was delivered on a VT 60 -sec schedule for $20 \mathrm{~min}$ or until the subject refused to consume it. At the end of this 20-min session (or $10 \mathrm{~min}$ after consumption of the last reinforcer), the subjects were given a $0.5-\mathrm{ml} / \mathrm{kg}$ intraperitoneal (i.p.) injection of $0.6-\mathrm{M}$ lithium chloride $(\mathrm{LiCl})$ and then returned to their home cages. On the second day of each cycle, the other reinforcer was delivered on a VT 60 . sec schedule for $20 \mathrm{~min}$, but at the end of the session, the subjects were simply returned to their home cages. For half the subjects, an aversion was established to the sucrose reinforcer but not to food pellets; for the remaining subjects, food pellets were conditioned but not the sucrose. Designation of the to-be-conditioned reinforcer was counterbalanced across responses. Throughout this phase of the experiment, access to the response manipulanda was prevented. Also, the doors of the sound-attenuating shells were open during the last three cycles to permit observation of consumption; the room was illuminated with red light.

On the day following the final cycle of aversion training, two 10-min extinction tests were administered to all subjects. In the first test, the subjects were permitted a choice between the lever and the chain; in the second test, the nose-poke and the handle-pull responses were simultaneously available. In this way, the order of testing extensively and moderately trained responses was balanced; half the animals were tested first with the two extensively trained responses and the remaining animals were tested first with the moderately trained responses. Responding was never reinforced during these tests. The next day, each response was tested separately for $4 \mathrm{~min}$. The order of testing was: nose-poke, chain-pull, leverpress, and handle-pull.

Finally, the subjects were tested for their consumption of the reinforcers in order to assess the effectiveness of the aversion treatment. The subjects were placed in the operant chambers and, after $1 \mathrm{~min}$, a pellet was delivered. An observer recorded whether or not the reinforcer was consumed within $5 \mathrm{~min}$ of its delivery. This test was repeated half an hour later with the sucrose reinforcer. The response manipulanda were not accessible to the animals during these consumption tests.

\section{Results and Discussion}

Responding reinforced extensively with cherry-flavored pellets increased over the course of VI training, reaching a terminal rate of 13.7 responses/min. This rate of responding remained unchanged when the cherry pellets were replaced with either sucrose or food pellets. In the second session of VI training following the shift in rein- forcer identity, the rate of responding for the extensively trained responses (13.7 responses/min) was slightly, but significantly, higher than the moderately trained pair (10.5 responses/min) [Wilcoxon $T(16)=25, p<.05$ ]. Although the pellet reinforcer supported slightly more responding than the sucrose reinforcer (12.1 and 11.1 responses/min, respectively), the difference did not reach statistical significance. There were no other significant differences.

Consumption of the poisoned reinforcer declined rapidly over the course of flavor-aversion conditioning. By the final cycle, no animal consumed any poisoned reinforcer, but all subjects ate all of the nonpoisoned reinforcers.

The data of primary interest are the response rates during the choice extinction tests. Figure 1 displays, in blocks of $2 \mathrm{~min}$, the results of those tests separated according to whether responding had been extensively (left panel) or moderately (right panel) pretrained with the cherry pellet reinforcer. The data are collapsed across response and reinforcer identity because these variables produced no reliable effect during extinction testing. It is clear that regardless of the amount of previous experience with the response, there was a clear preference for the response whose most recent reinforcer was still valuable. Responding was significantly depressed on the manipulandum whose reinforcer had been paired with a toxin in both the moderate $[T(16)=7, p<.01]$ and the extended pretraining conditions $[T(16)=23, p<.02]$. A similar pattern emerged in the single response tests, but the levels of responding were in general lower and the differences were not significant.

The results of the consumption tests given 2 days after the choice extinction tests revealed that the aversion treatment had been extremely successful. No animal consumed the poisoned reinforcer, but all animals ate the nonpoisoned reinforcer.

This study demonstrates that extended training of a response does not remove its ability to become associated with new reinforcers, even when features of the training procedure other than reinforcer identity remain unchanged. Indeed, in the present experiment, quite minimal exposure to the differential reinforcement conditions was sufficient to establish substantial response-reinforcer associations, whether or not the response had been subjected to extended pretraining. These results are consistent with the view that response-reinforcer associations influence instrumental performance throughout the course of learning.

The primary intention of this experiment was simply to identify some continued sensitivity of extensively trained responses to new information about the reinforcer. However, comparison of the two panels in Figure 1 suggests that reinforcer devaluation may have had a more substantial effect on responses that had not received extensive training with cherry pellets prior to the introduction of differential reinforcement. The difference between response rates for the devalued and nondevalued responses was significantly greater in the case of the moderately 


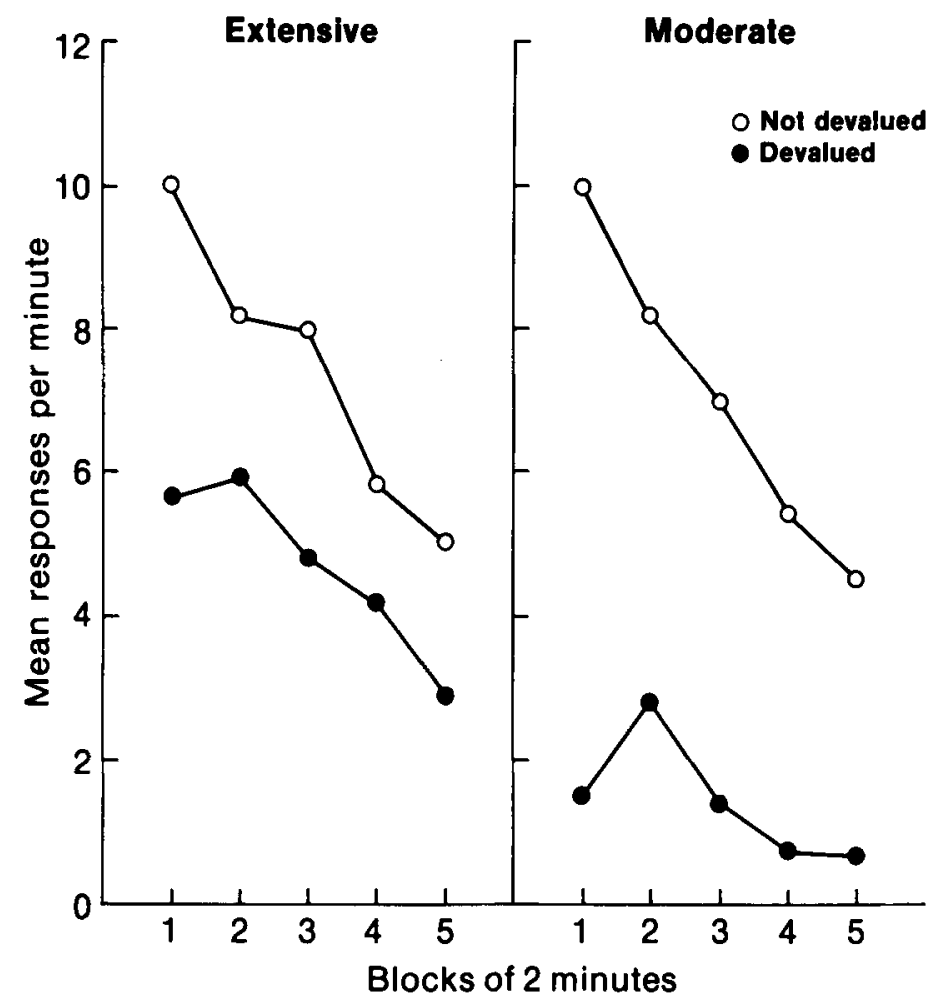

Figure 1. Experiment 1: Mean responses per minute during the choice extinction tests. An aversion had been conditioned to one reinforcer (solid symbols) but not to the other (open symbols). Responding that had undergone extensive pretraining with the cherry-flavored pellets is shown on the left, and that given moderate pretraining is shown on the right.

trained behaviors $[T(16)=29, p<.05]$. This might be interpreted to mean that although the prior reinforcement with cherry pellets did not prevent learning about the new reinforcers, it did reduce its magnitude. However, there are two reasons to be cautious about drawing such a conclusion. First, the prior training may not attenuate subsequent learning but, instead, provide both responses with a common associate also capable of supporting responding. A continued response-cherry pellet association might be capable of supporting performance even of the response whose differential reinforcer had been devalued. That interpretation correctly anticipates that the effect of prior training should be evidenced primarily by greater continued performance of the devalued response. Second, terminal levels of performance were reliably greater for the extensively trained responses. Such baseline differences complicate a comparison of differences in the magnitude of the effect of reinforcer devaluation across training conditions. The aim of Experiment 2 was to eliminate the complication of baseline differences in evaluating the sensitivity to reinforcer devaluation of responses that had received different amounts of training.

\section{EXPERIMENT 2}

This experiment attempted to compare directly the degree of sensitivity to reinforcer devaluation shown by moderately and extensively trained behaviors. Colwill and
Rescorla (1985a) found sensitivity after both moderate and extensive training, but were unable to make such a direct comparison because of differences in the levels of responding at the end of instrumental training. As was also the case in Experiment 1, the greater level of responding shown after extensive training precluded a meaningful comparison of the magnitudes of decrement produced by a devaluation operation. The present experiment used a design that guaranteed the same terminal level of performance for behaviors reinforced extensively or moderately. This was accomplished by following the very same responses with two reinforcers but varying the relative frequency of those reinforcers. Each animal was trained to make two different instrumental responses (leverpressing and chain-pulling). Each response was reinforced with two different reinforcers (liquid sucrose and food pellets). However, one response was extensively trained with sucrose and moderately trained with food pellets; the other response received moderate training with sucrose but extensive training with food pellets. Following this training, one of the reinforcers was paired with a toxin, and then all animals were given access to the lever and chain in a choice extinction test. A schematic of the design is shown in Figure 2.

The critical feature of this design is that the same response receives different amounts of training with two different reinforcers. Consequently, we can compare the effects of devaluing the extensive or moderate reinforcer 


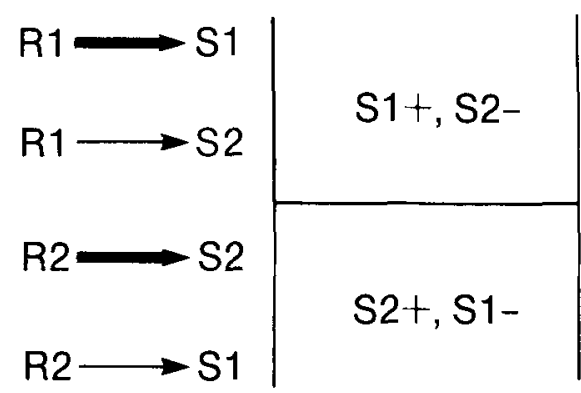

R1 vs R2

Figure 2. Design of Experiment 2: R1 and R2 are instrumental responses, leverpressing and chain-pulling counterbalanced across animals. Narrow arrow indicates moderate training and wide arrow indicates extensive training. S1 and S2 are sucrose liquid and food pellets. + and - represent the presentation or not of $\mathrm{LiCl}$.

on the same response beginning with the same terminal level of performance. The question of interest was whether that response would be more seriously affected by a devaluation of the reinforcer with which it received extensive training or by a devaluation of the reinforcer with which it received moderate training. A greater impact of the devaluation of the extensive reinforcer would suggest continued response-reinforcer learning with continued training. A greater impact of the devaluation of the moderate reinforcer would suggest a reduction in the role of response-reinforcer learning with continued training.

\section{Method}

Subjects. The subjects were 16 experimentally naive Holtzmanderived male rats about 100 days old at the start of the experiment. They were maintained in individual cages at $80 \%$ of their freefeeding weights. Water was available on an ad-lib schedule.

Apparatus. The apparatus consisted of eight Skinner boxes identical to those used in Experiment 1, except that they were not equipped with the nose-poke and handle-pull manipulanda.

Procedure. All subjects received one session of magazine training, consisting of $1045-\mathrm{mg}$ food pellets (Formula A: P. J. Noyes) followed by 10 presentations of $0.3 \mathrm{ml}$ of $8 \%$ sucrose liquid on a VT 60 -sec schedule. During this phase, the response manipulanda were not accessible to the animals

Following magazine training, all subjects were trained to press the lever and pull the chain. As in the previous experiment, only one manipulandum was available in any training session. Initially, each response was programmed to produce a reinforcer. Chainpulling was reinforced on Day 1 and leverpressing on Day 2, both with the pellet reinforcer. On Days 3 and 4 , leverpressing and chainpulling, respectively, were reinforced with sucrose. These sessions terminated after 25 reinforcers had been earned. Each response was then reinforced on a VI 30-sec schedule. Two 20-min training sessions were given each day for 2 days. On Day 1 , leverpressing and chain-pulling were reinforced with sucrose; on Day 2, these responses were reinforced with pellets.

Subjects continued to receive two 20-min sessions per day, one with the lever and one with the chain-pull, but responding was now reinforced on a VI 60 -sec schedule. For 14 days, one of these responses was reinforced with sucrose and the other with pellets. Training of a response with its moderate reinforcer was intermixed with this extensive training: After the 4th and 10th session of this extensive training, the response-reinforcer relations were switched for one 20 -min training session with each response. Thus, each response was trained moderately with one of the reinforcers and extensively with the other reinforcer. Moreover, the reinforcer that was used to train one of the responses extensively was used to train the other response moderately. The consequence of this procedure was to equate exposure to the reinforcers and to equate the levels of responding of a response that had undergone different amounts of training with its reinforcers.

After completion of response training, an aversion was conditioned to one of the reinforcers. The procedure for conditioning the reinforcers was identical to that described for Experiment 1 . For half the subjects, an aversion was established to the sucrose reinforcer; for the remaining subjects, food pellets were conditioned. Designation of the to-be-conditioned reinforcer was counterbalanced across responses. This training was conducted over six 2-day cycles. Throughout this phase of the experiment, access to the response manipulanda was prevented. Also, the doors of the sound-attenuating shells were open during the last four cycles to permit observation of consumption; the room was illuminated with red light.

On the day following the final cycle of aversion training, one 20 -min extinction test with both lever and chain available was administered to all subjects. Responding was never reinforced during this test.

Finally, to assess the effectiveness of the aversion treatment, the subjects were tested for their consumption of the reinforcers. The subjects were placed in the operant chambers and, after $1 \mathrm{~min}$, a pellet was delivered. Five minutes later, an observer recorded whether or not the reinforcer had been consumed. This test was repeated $30 \mathrm{~min}$ later with the sucrose reinforcer. The response manipulanda were not accessible to the animals during these consumption tests.

\section{Results and Discussion}

Responding increased over the course of VI training, reaching a terminal rate of 11.1 responses $/ \mathrm{min}$. There were no significant differences between the rates of chainpulling and leverpressing in the final sessions of training with either the moderate or extensive reinforcer. However, in these sessions, the pellet reinforcer supported a higher level of responding than the sucrose reinforcer, both in the moderate condition (10.8 vs. 9.2 responses $/ \mathrm{min}$ ) and in the extended condition (12.9 vs. 9.3 responses $/ \mathrm{min})[T \mathrm{~s}(16) \leq 27.5, p \mathrm{~s}<.05]$. These differences are not surprising because casual observations have indicated that the time taken to consume the sucrose reinforcer may be considerably longer than that taken to consume the pellet reinforcer. Such a difference in consumption time affects the time available for responding.

Consumption of the poisoned reinforcer declined rapidly over the course of flavor-aversion conditioning. By the final cycle, no animal consumed any poisoned reinforcer, but all subjects ate all of the nonpoisoned reinforcers.

The data of primary interest are the response rates during the choice extinction test. Figure 3 displays, in blocks of $4 \mathrm{~min}$, the results of that test separated according to whether responding had been moderately or extensively trained with the reinforcer that was subsequently devalued. The data are collapsed across response and reinforcer identity because these variables produced no reliable effect during extinction testing. It is clear that responding was substantially more depressed following poisoning of its more frequent reinforcer than following poisoning of the less frequent reinforcer $[T(16)=25, p<.05]$. 


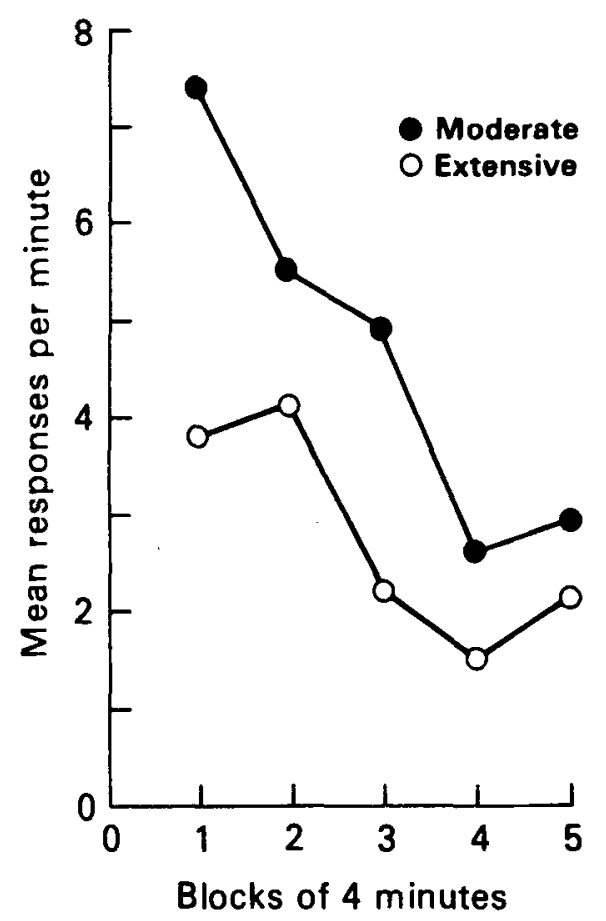

Figure 3. Experiment 2: Mean responses per minute during the choice extinction test shown separately for the response whose moderate reinforcer was devalued (solid symbols) and for the response whose extensive reinforcer was devalued (open symbols).

The results of the consumption tests given the day after the extinction test revealed that the aversion treatment had been extremely successful. No animal consumed the poisoned reinforcer, but all animals ate their nonpoisoned reinforcer.

The results of this experiment suggest that performance is influenced more strongly by the current value of the reinforcer that the response had earned extensively than by the current value of the reinforcer earned only moderately. This conclusion is especially compelling because the design permitted a direct comparison of the effects of different amounts of training without the contaminating problem of differences in levels of responding. Equivalent levels of responding after various amounts of training with a reinforcer were accomplished by following the same response by two reinforcers, one extensively and one moderately. Thus, the outcome of this study is consistent with the view that the major consequence of repeated reinforcement is to increase the contribution of response-reinforcer associations to instrumental performance. It does not accord with a view that the role of response-reinforcer associations declines with extensive use of a reinforcer.

\section{GENERAL DISCUSSION}

The results of these experiments agree with the conclusion drawn by Colwill and Rescorla (1985a) that extended training does not make a response insensitive to subsequent changes in the value of its reinforcer. Indeed, the findings of Experiment 2 indicate that additional train- ing enhances that sensitivity, suggesting that instrumental performance remains dependent on response-reinforcer associations after extended training. Moreover, Experiment 1 explicitly demonstrated that even an extensively trained response becomes associated with new reinforcers that are made contingent upon it. Thus, these experiments provide no support for the view that, with repeated practice, instrumental responding becomes independent of its reinforcer.

Of course, it remains possible that after extensive training, responding is not normally controlled by the responsereinforcer association. It might be that some feature of our testing procedure induced sensitivity of the response to reinforcer devaluation. For instance, a testing procedure that provided the opportunity to choose between different responses during extinction may have encouraged the use of such associations. However, Colwill and Rescorla (1985a) reported that extensively trained behaviors are sensitive to reinforcer devaluation even in the absence of a choice test. Moreover, it is not clear how one would rule out the possibility that response-reinforcer associations become latent until some intrusive test procedure is adopted. But it is clear that these associations persist and continue to govern performance with extensive training.

It is worth noting that the present designs may provide generally powerful techniques for investigating the nature of instrumental learning. Experiment 1 used a procedure in which two responses were pretrained with a common reinforcer prior to being reinforced with different outcomes. The combination of this treatment with subsequent reinforcer devaluation has two important advantages.

First, the pretraining allows one to explore subtle changes in information about the reinforcer under conditions that avoid the high variability attendant upon initial response shaping. As already noted, considerable response-reinforcer learning was revealed with as few as $\mathbf{4 0}$ differential reinforcers following each response. The magnitude of that effect suggests the possibility that very few conjunctions indeed may be required to establish response-reinforcer associations.

Second, the devaluation procedure gives Experiment 1 an advantage over a historically important class of experiments in which the reinforcer magnitude is changed in the course of learning (e.g., Crespi, 1942; Zeaman, 1949). In those experiments, learning about the reinforcer was inferred from a sudden shift in performance that resulted from a change in magnitude of the reinforcer. In Experiment 1, a shift was made between two similarly valued reinforcers. Consequently, there was no rapid adjustment in performance from which to infer learning about the reinforcer. Nevertheless, subsequent devaluation of the reinforcer revealed substantial encoding of the reinforcer identity. This observation highlights the limitations of immediate performance shifts as measures of the degree of reinforcer encoding. They may fail to detect learning about reinforcers in the absence of substantial shifts in value. By contrast, the present devaluation technique is sensitive to changes in response outcomes other than their total reinforcing power. 
The design used in Experiment 2 may also be generally useful for analyzing instrumental learning. Training the same response with two different reinforcers allowed us to compare the degree of learning about a reinforcer under variations in parameter values that would ordinarily result in quite different levels of performance. In Experiment 2 , that technique was used to compare learning after amounts of training that would otherwise have yielded incomparably different baselines of behavior. However, it seems likely that an analogous procedure could be used to investigate the degree of response-reinforcer learning when other parameters of reinforcement are varied. For instance, it is of interest to ask whether variations in the magnitude or delay of reinforcement would produce different amounts of response-reinforcer learning. By using the procedure of Experiment 2, one could compare directly the effects of devaluing either the immediate or the delayed reinforcer on responding without facing the problem of differences in predevaluation performance.

Consequently, the present experiments are of interest not only because they allowed us to evaluate further a particular hypothesis about instrumental learning, but also because they incorporated design features that may be of general use in studying instrumental learning.

\section{REFERENCES}

AdAms, C. D., \& Dickinson, A. (1981). Actions and habits: Variations in associative representations during instrumental learning. In
N. E. Spear \& R. R. Miller (Eds.), Information processing in animals: Memory mechanisms (pp. 143-165). Hillsdale, NJ: Erlbaum.

AllPORT, G. W. (1937). Personality: A psychological interpretation. New York: Holt.

Colwill, R. M., \& Rescorla, R. A. (1985a). Instrumental responding remains sensitive to reinforcer devaluation after extensive training. Journal of Experimental Psychology: Animal Behavior Processes, 11, $520-536$

Colwill, R. M., \& Rescorla, R. A. (1985b). Post-conditioning devaluation of a reinforcer affects instrumental responding. Journal of $E x$ perimental Psychology: Animal Behavior Processes, 11, 120-132.

ColwiLl, R. M., \& Rescorla, R. A. (1986). Associative structures in instrumental learning. In G. H. Bower (Ed.), The psychology of learning and motivation (Vol. 20, pp. 55-104). New York: Academic Press.

CresPI, L. P. (1942). Quantitative variation of incentive and performance in the white rat. American Journal of Psychology, 55, 467-517.

GiLhousen, H. C. (1933). Fixation of excess distance patterns in the white rat. Journal of Comparative Psychology, 16, 1-24.

Kimble, G. A., \& Perlmuter, L. C. (1970). The problem of volition. Psychological Review, 77, 361-384.

MurPhy, G. (1947). Personality: A biosocial approach to origins and structure. New York: Harper.

Tolman, E. C. (1933). Sign-Gestalt or conditioned reflex? Psychological Review, 40, 246-255.

Tolman, E. C. (1948). Cognitive maps in rats and men. Psychological Review, 55, 189-208.

ZEAMAN, D. (1949). Response latency as a function of the amount of reinforcement. Journal of Experimental Psychology, 39, 446-483.

(Manuscript received April 27, 1987; revision accepted for publication September 22, 1987.) 